\title{
Lower serum expression of miR-181c-5p is associated with increased plasma levels of amyloid-beta 1-40 and cerebral vulnerability in normal aging
}

\author{
Marta Manzano-Crespo ${ }^{1}$, Mercedes Atienza ${ }^{1,2}$ and Jose L. Cantero ${ }^{1,2^{*}}$
}

\begin{abstract}
Background: Previous studies have shown that expression levels of miR-181c are downregulated by amyloid- $\beta$ (A $\beta$ ) deposition and chronic cerebral hypoperfusion, both factors largely associated with the development of AD. Moreover, reduced 2-[18F]fluoro-2-deoxy-D-glucose (FDG)-PET brain metabolism and volume loss of regions of the medial temporal lobe have been generally recognized as hallmarks of AD. Based on this evidence, we have here investigated potential associations between serum levels of miR-181c-5p and these AD signatures in asymptomatic elderly subjects.

Methods: Ninety-five normal elderly subjects underwent clinical, cognitive, structural MRI, and FDG-PET explorations. Serum expression levels of miR-181c-5p and plasma A $\beta$ concentrations were further analyzed in this cohort. Regression analyses were performed to assess associations between serum miR-181c-5p levels and cognitive functioning, plasma $A \beta$, structural and metabolic brain changes.

Results: Decreased serum expression of miR-181c-5p was associated with increased plasma levels of $A \beta_{1-40}$, deficits in cortical glucose metabolism, and volume reduction of the entorhinal cortex. No significant associations were found between lower miR-181c-5p levels and cognitive deficits or cortical thinning.

Conclusions: These findings suggest that deregulation of serum miR-181c-5p may indicate cerebral vulnerability in late life.
\end{abstract}

Keywords: Aging, Alzheimer's disease, Blood biomarkers, microRNAs, Amyloid-beta, FDG-PET, Entorhinal cortex

\section{Background}

Aging is an extremely complex and inexorable process characterized by gradual accumulation of biological damage that leads to impaired cell homeostasis, decreased organ mass, and loss of functional reserve of the body's systems [1]. Aging is not exactly a disease but has the capacity to increase the risk for developing a wide range of chronic non-communicable conditions [2]. Most importantly, experimental manipulations aimed at slowing down aging have shown to improve survival,

\footnotetext{
* Correspondence: jlcanlor@upo.es
'Laboratory of Functional Neuroscience, Pablo de Olavide University, Ctra. de

* Correspondence: jlcanlor@upo.es
'Laboratory of Functional Neuroscience, Pablo de Olavide University, Ctra. de Utrera Km 1, 41013 Seville, Spain

${ }^{2}$ CIBERNED, Network Center for Biomedical Research in Neurodegenerative Diseases, 28031, Madrid, Spain
}

delay disease onset, and reduce the rate of aging-related chronic disorders [3-5], suggesting that intervening in aging could prevent chronic diseases and extend healthspan [6, 7].

With the unprecedented aging population, the burden of dementia has increased substantially. Alzheimer's disease (AD), the most common cause of dementia among older people, is rapidly becoming a major public health problem in developed countries. Identifying $\mathrm{AD}$ vulnerability in asymptomatic individuals is a challenging endeavor due to the remarkable variability in aging phenotypes, lifestyles, and environmental exposures. Considering that $\mathrm{AD}$ is becoming a healthcare burden of epidemic proportion, there is an urgent need for identifying biomarkers of susceptibility to developing $\mathrm{AD}$ before cognitive symptoms arise. 
Ideally, these biomarkers should be minimally invasive and cost-effective, uncover aspects of AD pathology, and correlate with subclinical changes in $\mathrm{AD}$-related brain regions.

MicroRNAs (miRNAs) are small, non-coding RNAs that regulate gene expression and protein synthesis by inducing degradation or suppressing translation of the target messenger RNA (mRNA) [8]. Evidence suggests that miRNAs are released from neurons into the bloodstream, where they are highly stable over time and can be detected non-invasively $[9,10]$. Consequently, blood miRNA expression levels may reveal dysregulation of brain-enriched miRNAs due to different aspects of AD pathogenesis, including inflammation, lipid metabolism, oxidative stress, and proteinopathy [11]. Many recent studies have identified blood miRNA expression profiles in AD patients, suggesting that they are able to differentiate clinical AD from normal aging with reasonable accuracy [12-18]. However, there is a lack of research on AD-related miRNAs combined with neuroimaging markers in asymptomatic individuals, who may be selected for timely interventions to reduce the risk of developing dementia.

Previous studies have revealed that miR-181c regulates amyloid- $\beta$ (A $\beta)$ deposition through changes in membrane ceramide levels and lipid rafts [19]. Expression of miR-181c, especially miR-181c-5p, has found to be correlated with increased $A \beta$ levels [20], and deregulated in brain [21-23] and blood of AD patients [12, 17, 24, 25]. Based on this evidence, we have here investigated potential associations between serum expression levels of miR-181c-5p and plasma $A \beta$, cognitive and brain changes in asymptomatic elderly subjects. Our hypothesis is that lower levels of miR-181c-5p should parallel changes in plasma $A \beta$ concentrations, deficits in $A D-$ related brain regions, and impaired cognition, likely revealing greater cerebral vulnerability in late life.

\section{Methods \\ Participants}

Ninety-five normal elderly subjects, recruited from senior citizens associations, health screening programs, and hospital outpatient services, participated in the study. They showed normal cognitive performance in the neuropsychological tests relative to appropriate reference values for age and education level. Individuals with medical conditions and/or history of conditions that may affect brain structure or function (e.g., neurodegenerative diseases, stroke, head trauma, hydrocephalus, and/or intracranial mass) were not included in the study. All subjects showed a global score of 0 (no dementia) in the Clinical Dementia Rating (CDR), normal global cognitive status in the Mini Mental State Examination (MMSE) (scores $\geq 26$ ), and normal independent function -assessed by the Spanish version of the Interview for Deterioration in Daily Living Activities [26]. Depression was excluded (scores $\leq 5$ ) by the Geriatric
Depression Scale [27]. All participants gave informed consent to the experimental protocol approved by the Ethical Committee for Human Research at the University Pablo de Olavide according to the principles outlined in the Declaration of Helsinki.

\section{Neuropsychological assessment}

A neuropsychological battery covering memory, executive functioning and language was administered to all participants. Subjective memory was evaluated with the Memory Functioning Questionnaire (MFQ), while objective memory was assessed with the Free and Cued Selective Reminding Test (FCSRT). The Tower of London (TOL) and the Boston Naming Test (BNT) were administered to evaluate executive function and naming, respectively.

Total RNA isolation and miRNA quantification by qRT-PCR Venous blood samples were collected in serum-gel $9 \mathrm{ml}$ tubes (Sarstedt S-Monovette ${ }^{\circledast}$ ) after overnight fasting, centrifuged at $1900 \mathrm{~g}$ for $10 \mathrm{~min}\left(4^{\circ} \mathrm{C}\right)$ followed by a second centrifugation at $16000 \mathrm{~g}$ for $5 \mathrm{~min}\left(4^{\circ} \mathrm{C}\right)$, and stored at $80^{\circ} \mathrm{C}$. Total RNA was isolated from $200 \mu \mathrm{l}$ aliquots of serum in each sample using the miRNeasy Serum/Plasma kit (Qiagen, Germany). Synthetic miRNA cel-miR-39-3p $\left(3.5 \mu \mathrm{l}, 1.6 \times 10^{8} \mathrm{copies} / \mu \mathrm{l}\right)$ was used for sample-to-sample normalization in RNA isolation. Both concentration (range: $8-25 \mathrm{ng} / \mu \mathrm{l}$ ) and purity (260/280 ratio: $1.5-2)$ of RNA were measured using the NanoDrop 2000 spectrophotometer (Thermo Fisher Scientific), and RNA samples were stored at $-80^{\circ} \mathrm{C}$ until use.

RNA was reverse transcribed with the $\operatorname{TaqMan}^{\oplus}$ miRNA Reverse Transcription Kit and miRNA-specific stem-loop primers (Applied Biosystems), according to the manufacturer's instructions. The reverse transcription (RT) reaction $(15 \mu \mathrm{l})$ was composed of $5 \mu \mathrm{l}$ of RNA (at a concentration of $10 \mathrm{ng} / \mu \mathrm{l}), 7 \mu \mathrm{l}$ of master mix, and $3 \mu \mathrm{l}$ of miRNA-specific stem-loop RT primer. Reactions were performed in triplicate for the miR-181c-5p, incubated at $16{ }^{\circ} \mathrm{C}$ for $30 \mathrm{~min}$, $42^{\circ} \mathrm{C}$ for $30 \mathrm{~min}, 85^{\circ} \mathrm{C}$ for $5 \mathrm{~min}$, and then maintained at $4{ }^{\circ} \mathrm{C}$. The complementary DNA (cDNA) products were stored at $-80^{\circ} \mathrm{C}$ until further analysis.

For quantitative real-time PCR (qRT-PCR), the reaction $(20 \mu \mathrm{l})$ for each sample was composed of $1.33 \mu \mathrm{l}$ of cDNA products, $1 \mu \mathrm{l}$ of TaqMan ${ }^{\odot}$ Small RNA Assay (Applied Biosystems), $10 \mu \mathrm{l}$ of TaqMan ${ }^{\bullet}$ Universal PCR Master Mix II (2X), and $7.67 \mu$ l of nuclease-free water. Each PCR reaction was performed in triplicate with a StepOnePlus real-time PCR system (Applied Biosystems) on a 96-well plate, and incubated at $4{ }^{\circ} \mathrm{C}$ for $2 \mathrm{~min}, 95^{\circ}$ for 10 min, followed by 40 cycles at $95^{\circ} \mathrm{C}$ for $15 \mathrm{~s}$ and $60{ }^{\circ} \mathrm{C}$ for $1 \mathrm{~min}$. Before analysis, individual cycle threshold $(\mathrm{Ct})$ values were examined to detect outliers. Replicas showing $\mathrm{Ct}$ differences higher than 0.5 were systematically repeated. Validated $\mathrm{Ct}$ values were averaged and 
normalized to the mean of the spiked cel-miR-39-3p in each sample (Additional file 1: Figure S1). The relative expression of miR-181c-5p was calculated with the fold change method [28], and used for statistical purposes. We measured the amplification efficiency of the qPCR reactions based on the slope of the standard curve. PCR efficiency was 97\% (for cel-miR-39-3p) and 96\% (for miR-181c-5p). TaqMan miRNA Assays (hsa-miR-181c$5 p$ and cel-miR-39-3p) for qRT-PCR experiments were purchased from Applied Biosystems. RNA isolation and miRNA quantification methods have been reported elsewhere [29].

\section{Plasma $A \beta$ levels}

Blood samples for miRNA quantification and $A \beta$ analysis were collected at the same time in all participants. Plasma $A \beta$ levels were determined by a double-antibody sandwich ELISA (human $A \beta_{1-40}$ and high sensitive $A \beta_{1-42}$, Wako Chemicals, Tokyo, Japan). Briefly, venous blood samples were collected after overnight fasting in $10 \mathrm{~mL} \mathrm{K2-ethyl-}$ enediaminetetraacetic acid (EDTA) coated tubes (BD Diagnostics), and immediately centrifuged $(1989 \mathrm{~g})$ at $4{ }^{\circ} \mathrm{C}$ for $5 \mathrm{~min}$. Supernatant plasma was collected into polypropylene tubes containing $250 \mu \mathrm{L}$ of plasma mixed with $8.32 \mu \mathrm{L}$ of a protease inhibitor cocktail (cOmplete Ultra Tablets mini, Roche). Plasma samples were stored at $80^{\circ} \mathrm{C}$ and thawed immediately before assay.

Samples and standards were incubated overnight at $8{ }^{\circ} \mathrm{C}$ with antibodies specific for $\mathrm{A} \beta_{1-40}$ or $\mathrm{A} \beta_{1-42}$ peptides, and the wells were read for absorption at $450 \mathrm{~nm}$ on a Victor 3 system (PerkinElmer, Waltham, MA), according to the manufacturer's instructions. Plasma $A \beta$ levels were measured in duplicate $(50 \mu \mathrm{L})$, and the average of the two measurements $(\mathrm{pg} / \mathrm{ml})$ was used for statistical purposes. Both inter-assay and intra-assay coefficients of variation were below $10 \%$. The detection limit for these assays was $1.04 \mathrm{pg} / \mathrm{ml}$ for $\mathrm{A} \beta_{1-40}$ and $0.54 \mathrm{pg} / \mathrm{ml}$ for $\mathrm{A} \beta_{1-42}$.

\section{MRI and FDG-PET acquisition}

Structural brain images were acquired on a Philips Achieva $3 \mathrm{~T}$ MRI scanner equipped with a body transmit coil and an 8-channel receive head coil (Philips, Best, Netherlands). T1-weighted magnetization-prepared rapid gradient echo (MP-RAGE) cerebral images were obtained for each participant. Acquisition parameters were empirically optimized for gray/white matter contrast (repetition time $=2300 \mathrm{~ms}$, echo time $=4.5 \mathrm{~ms}$, flip angle $=8^{\circ}$, matrix dimensions $=320 \times 320$, voxel size $=0.8 \mathrm{~mm}$ isotropic resolution, no gap between slices, acquisition time $=9.1$ $\mathrm{min})$. Head motion was controlled using a head restraint system and foam padding around the subject's head.

FDG-PET brain images were acquired on a wholebody PET-TAC Siemens Biograph 16 HiREZ scanner (Siemens Medical Systems, Germany). Subjects fasted for at least $8 \mathrm{~h}$ before PET examination, and they were scanned at the same time of the day $(8: 00-9: 00 \mathrm{am})$. Intravenous lines were placed $10-15 \mathrm{~min}$ before tracer injection of a mean dose of $370 \mathrm{MBq}$ of 2-[18F]fluoro-2deoxy-D-glucose (FDG). PET scans lasted approximately $30 \mathrm{~min}$. All PET images were corrected for attenuation, scatter and decay, smoothed for uniform resolution, and reconstructed with $2.6 \times 2.6 \times 2 \mathrm{~mm}$ voxel resolution using back-projection filters. MRI-based correction of FDG-PET data for partial volume effects was performed with the PMOD software v3.208 (PMOD Technologies Ltd., Switzerland) using the Müller-Gartner approach.

\section{Estimation of surface-based cortical thickness and cortical glucose metabolism}

MRI data were processed using the analysis pipeline of Freesurfer v6.0 (https://surfer.nmr.mgh.harvard.edu/) that involves intensity normalization, registration to Talairach, skull stripping, white matter (WM) segmentation, tessellation of the WM boundary, and automatic correction of topological defects [30]. Pial surface misplacements and erroneous WM segmentation were manually corrected on a slice-by-slice basis to enhance the reliability of cortical thickness measurements. Cortical thickness maps were smoothed using non-linear spherical wavelet-based de-noising schemes [31].

To map the FDG uptake onto cortical surfaces, we first co-registered individual FDG-PET images to T1weighted images using PMOD tools. Next, partial volume corrected cortical FDG-PET images were sampled onto the subject's cortical surface, transformed to the Freesurfer standard surface space, and smoothed with non-linear spherical wavelet-based de-noising schemes [31]. Finally, FDG activity assigned to each cortical surface vertex was normalized by the FDG activity of the entire cortex using an iterative vertex-based statistical method that excludes group-dependent vertices from calculation of global activity [32].

Anatomical ROIs of AD-related regions were created using a semiautomatic approach implemented in Freesurfer. Briefly, segmented brain images were parcellated into different brain regions according to the Destrieux atlas [33] and the Freesurfer automatic subcortical segmentation (aseg), respectively. This allowed us to obtain volume measurements of hippocampus, entorhinal cortex and parahippocampal gyrus, regions that have shown to be affected at early stages of AD [34].

\section{Statistical analyses}

We first assessed whether serum expression of miR181c-5p, plasma $A \beta$, and cognitive scores deviated from normality by applying the Kolmogorov-Smirnov test with the Lilliefors correction. Next, linear regression analyses were conducted to evaluate whether miR-181c- 
$5 p$ was associated with $A \beta$ levels $\left(A \beta_{1-40}\right.$ and $A \beta_{1-42}$, separately) and/or cognitive performance. Regression analyses were adjusted by age, sex and/or ApoE4 if these factors showed an statistically significant effect on any of the dependent variables. The alternative $A \beta$ peptide was included as a confounding factor to mitigate its potential influence on the relationship between the $A \beta$ peptide of interest and miR-181c-5p levels. We also investigated the impact of sex on regression coefficients by including the product of the dummy variable for sex and the miR$181 \mathrm{c}-5 \mathrm{p}$ as an interaction term in the regression model. These analyses were performed with SPSS v22 (SPSS Inc. Chicago, IL).

Vertex-wise linear regression analyses adjusted by age, sex and/or ApoE4 were further performed to determine whether serum expression levels of miR-181c-5p were associated with variations in cortical thickness/cortical glucose consumption. Results were corrected for multiple comparisons using a previously validated hierarchical statistical model [35]. This procedure first controls the family-wise error rate at the level of cluster by applying random field theory over smoothed statistical maps; and next controls the false discovery rate within significant clusters at the level of vertices over unsmoothed statistical maps. A significant cluster was defined as a contiguous set of cortical surface vertices that met the statistical threshold criteria ( $p<0.05$ after correction for multiple comparisons) and whose surface area was greater than $40 \mathrm{~mm}^{2}$.

Finally, linear regression analyses, adjusted by intracranial volume (ICV), as well as by age, sex and/or ApoE4 if necessary, were conducted to evaluate whether serum expression levels of miR-181c-5p were correlated with volume changes in the hippocampus, entorhinal cortex and/or parahippocampal gyrus. Regression analyses were also conducted for women and men separately. If at least one of the two groups showed significant results, we evaluated the effect of sex on regressions coefficients.

\section{Results}

Relationship between serum miR-181c-5p, plasma $A \beta$ levels, and cognitive performance

Serum miR-181c-5p, A $\beta$ levels and cognitive scores were normally distributed, allowing the use of parametric statistical tests. Table 1 shows demographic, neuropsychological, blood, and cerebral markers of the study sample. While cognitive performance was not associated with miR-181c-5p levels, regression analyses adjusted by age and $A \beta_{1-42}$ levels revealed that lower levels of miR-181c$5 \mathrm{p}$ were significantly correlated with higher $\mathrm{A} \beta_{1-40}(r=-$ $0.49, \mathrm{~F}_{3,91}=9.52, p=10^{-4}$ ) (Fig. 1a), but not with $\mathrm{A} \beta_{1-42}$ concentrations (Fig. 1b). When these analyses were segregated by sex (Additional file 2: Figure S2A-B), $\mathrm{A} \beta_{1-40}$ was related to mir-181c-5p levels only in women $(r=-0.37$,
Table 1 Demographic, neuropsychological, blood, and cerebral markers of the study sample

\begin{tabular}{|c|c|}
\hline & Sample $(N=95)$ \\
\hline Age & $68.8 \pm 4(62-78)$ \\
\hline Sex (M/W) & $54 / 41$ \\
\hline ApoE $\varepsilon 4$ (yes/no) & $21 / 74$ \\
\hline CDR & 0 \\
\hline MMSE & $29.3 \pm 1.2(26-30)$ \\
\hline MFQ & $36.8 \pm 10.5(16-66)$ \\
\hline FCSRT & $13.7 \pm 2(8-16)$ \\
\hline TOL & $413 \pm 139(132-824)$ \\
\hline Boston naming test & $52.3 \pm 4.5(40-59)$ \\
\hline miR-181c-5p (fold change) & $0.66 \pm 0.37(0.07-1.5)$ \\
\hline$A \beta_{1-40}(p g / m l)$ & $228.8 \pm 32.6(167.7-316)$ \\
\hline $\mathrm{A} \beta_{1-42}(\mathrm{pg} / \mathrm{ml})$ & $24 \pm 7.4(7.6-59)$ \\
\hline L hippocampus $\left(\mathrm{mm}^{3}\right)$ & $3140 \pm 328(2450-4043)$ \\
\hline R hippocampus $\left(\mathrm{mm}^{3}\right)$ & $3242 \pm 330(2487-4080)$ \\
\hline $\mathrm{L}$ entorhinal $\left(\mathrm{mm}^{3}\right)$ & $1144 \pm 124(862-1499)$ \\
\hline R entorhinal $\left(\mathrm{mm}^{3}\right)$ & $1670 \pm 199(1131-2162)$ \\
\hline L parahippocampal $\left(\mathrm{mm}^{3}\right)$ & $3355 \pm 635(2046-5411)$ \\
\hline R parahippocampal $\left(\mathrm{mm}^{3}\right)$ & $3154 \pm 539(2090-4488)$ \\
\hline
\end{tabular}

Results are expressed as mean \pm standard deviation for each group, range (min-max)

$M$ Men, $W$ Women, CDR Clinical dementia rating, MMSE Mini mental state examination, MFQ Memory functioning questionnaire, FCSRT Free and cued selective reminding test, TOL Tower of London, $L$ Left and $R$ Right

$\left.\mathrm{F}_{3,37}=4.51, p=10^{-2}\right)$. However, the regression coefficients did not significantly differ between women and men.

\section{Relationship between serum miR-181c-5p levels and volume of $A D$-related brain regions}

Table 1 contains mean volume of hippocampus, entorhinal cortex and parahippocampal gyrus of the study sample. Regression analyses adjusted by age and ICV showed that decreased serum levels of miR-181c-5p were significantly correlated with volume reduction of the entorhinal cortex $\left(r=0.59, \mathrm{~F}_{3,91}=16, p=10^{-7}\right)$. This relationship was mainly evident for the left entorhinal cortex $\left(r=0.57, \mathrm{~F}_{3,91}=14.6, p=10^{-7}\right)$ (Fig. 1c) although a trend to significance was also observed in the right entorhinal cortex (Fig. 1d). Changes in miR-181c-5p levels were unrelated to volume variations of either hippocampus or parahippocampal gyrus. Neither did we find sex differences in regression coefficients for any of the correlations performed with mir-181c-5p and AD-related brain regions (Additional file 2: Figure $\mathrm{S} 2 \mathrm{C}-\mathrm{H}$ ).

\section{Relationship between serum miR-181c-5p levels, cortical thickness, and cortical glucose consumption}

We next sought to investigate whether changes in serum levels of miR-181c-5p were correlated with variations in 

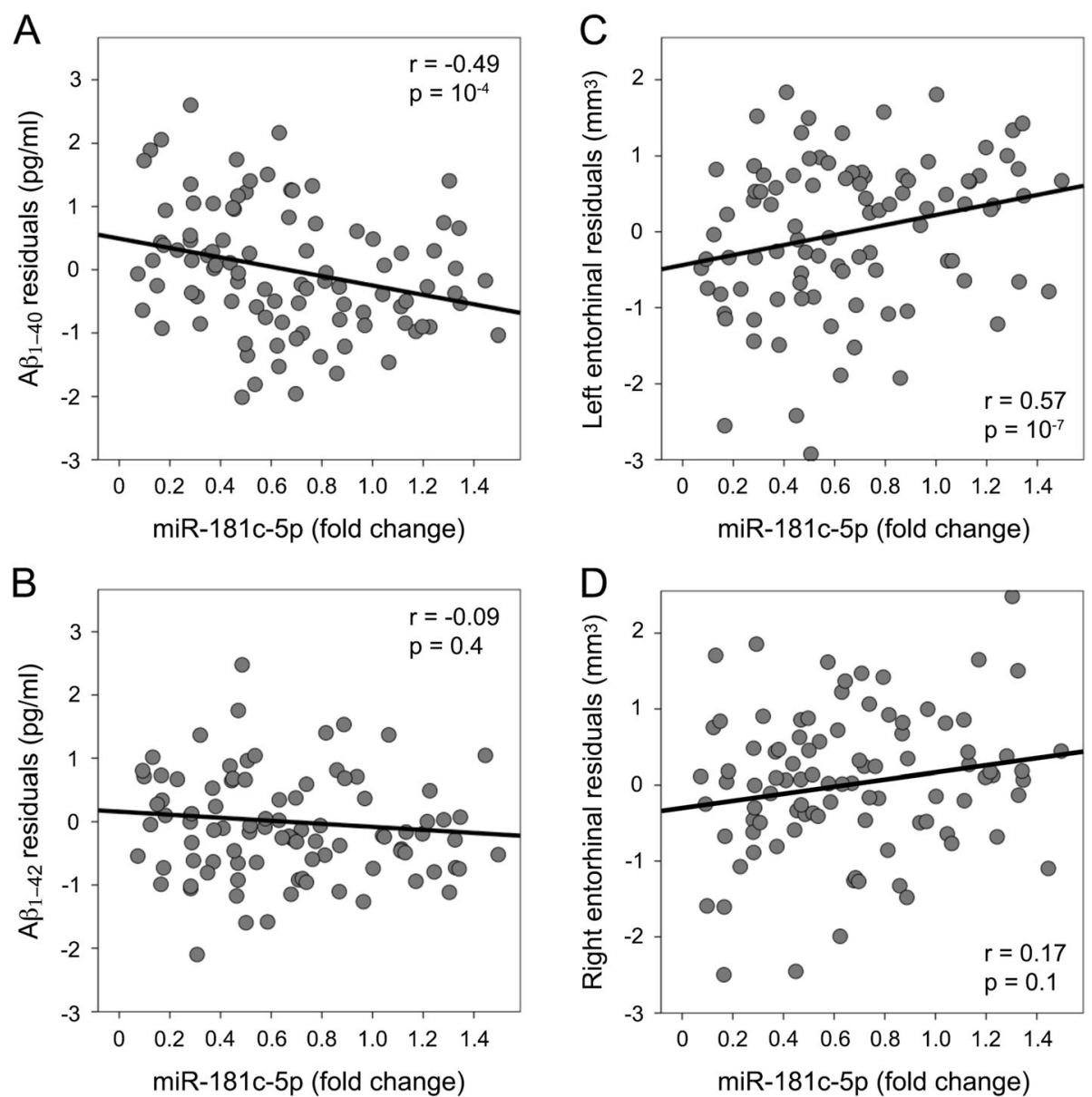

Fig. 1 Correlations between serum levels of miR-181c-5p and plasma A $\beta$ levels (a and $\mathbf{b}$ ), and volume of the entorhinal cortex (c and $\mathbf{d}$ ) Variables included in the scatter plots correspond to the standardized residuals obtained from linear regression analyses adjusted by age, $A \beta_{1-42}$ (in the case of $A \beta_{1-40}$ ), $A \beta_{1-40}$ (in the case of $A \beta_{1-42}$ ), and ICV (in the case of left and right entorhinal cortex). Note that only correlations with $A \beta_{1-40}$ and left entorhinal cortex yielded significance

cortical glucose consumption, after removing the effects of age and sex. Results indicated that decreased serum expression of miR-181c-5p was associated with lower glucose consumption in superior parietal regions bilaterally (left: $p=$ $10^{-4}$; right: $\left.p=10^{-6}\right)$, right inferior parietal areas $\left(p=10^{-6}\right)$, and right precuneus $\left(p=10^{-6}\right)$. Decreased FDG uptake within bilateral insula was also related to lower miR-181c-5p levels (left: $p=10^{-4}$; right: $p=10^{-5}$ ). Reduced serum levels of miR-181c-5p further predicted hypometabolism in the left entorhinal $\left(p=10^{-5}\right)$, right lingual gyrus $\left(p=10^{-4}\right)$, left superior frontal $\left(p=10^{-3}\right)$, and medial aspects of the right orbitofrontal cortex $\left(p=10^{-4}\right)$. These results are summarized in Table 2 and illustrated in Fig. 2. No significant associations were found between miR-181c-5p levels and changes in cortical thickness.

\section{Discussion}

There is an urgent need for inexpensive and reliable biomarkers able to identify individuals at greatest risk of developing AD. While blood molecules are ideally suited for this endeavor [41], lack of standardization of preanalytic conditions and poor reproducibility of results preclude their use as first-line diagnostic tools in clinical settings. miRNAs are stable circulating molecules that have shown to be deregulated in the prodromal and clinical phases of AD [42]. However, their usefulness for detecting at-risk subjects for developing $\mathrm{AD}$ remains to be determined. In the present study, we have shown that decreased serum expression of miR-181c-5p was associated with higher plasma $A \beta_{1-40}$ levels, deficits in cortical glucose metabolism, and volume reduction of the entorhinal cortex in asymptomatic elderly subjects. Overall, these results are likely revealing aging-related cerebral vulnerability linked to altered expression of miR-181c-5p, which has previously been associated with $A \beta$ regulation [19] and cerebral ischemia/hypoxia in both humans and animal models [43, 44], and has been found to be deregulated in the brain [21-23] and blood [12, 17, 24, 25] of AD patients. 
Table 2 Significant correlations between decreased serum levels of miR-181c-5p and lower cortical glucose uptake measured by FDG-PET

\begin{tabular}{llll}
\hline Cortical region & $\mathrm{CS}\left(\mathrm{mm}^{2}\right)$ & $r$ & $P$ \\
\hline L insula & 3891 & 0.29 & $10^{-4}$ \\
L entorhinal & 1588 & 0.3 & $10^{-5}$ \\
L superior parietal & 431 & 0.39 & $10^{-4}$ \\
L superior frontal & 245 & 0.31 & $10^{-3}$ \\
L anterior cingulate & 174 & 0.33 & $10^{-4}$ \\
R inferior parietal & 1531 & 0.38 & $10^{-6}$ \\
R insula & 839 & 0.37 & $10^{-5}$ \\
R medial orbitofrontal & 819 & 0.34 & $10^{-4}$ \\
R superior parietal & 583 & 0.35 & $10^{-6}$ \\
R lingual gyrus & 160 & 0.39 & $10^{-4}$ \\
R precuneus & 144 & 0.37 & $10^{-6}$ \\
\hline CS Cluster siz; it & &
\end{tabular}

CS Cluster size; it refers to the extent of significant correlation between serum levels of miR-181c-5p and cortical glucose uptake. $L$ Left and $R$ Right cortical hemisphere. Regression analyses were adjusted by age and sex. r: Pearson correlation coefficient; $\mathrm{p}$ : exact $p$-value (corrected for multiple comparisons)

Recent studies have assessed the role played by serum/ plasma expression of miR-181c as AD biomarker. Most of them evaluated the miR-181c-5p [12, 17, 25], except for one that employed the miR-181c-3p [24]. Here we found that decreased serum levels of miR-181c-5p were associated with higher concentrations of plasma $A \beta_{1-40}$. Previous studies have revealed that plasma $A \beta_{1-40}$ is increased in familial $\mathrm{AD}$ with mutations in the genes encoding presenilin or the amyloid precursor protein $[45,46]$, in patients with Down's syndrome $[47,48]$, and in first-degree relatives of $\mathrm{AD}$ patients who are at increased risk of developing the disease [49]. Moreover, elderly subjects with higher plasma $A \beta_{1-40}$ levels have shown slower processing and poorer memory together with bilateral thinning of the prefrontal cortex compared with those who had lower $A \beta_{1-40}$ levels, suggesting that higher plasma $A \beta_{1-40}$ may signal accelerated aging [50]. Plasma $A \beta_{1-40}$ levels have also shown to be elevated in cerebral small vessel disease, a frequent agingrelated pathology that contributes to the development of dementia [51, 52]. Recent evidence indicated that miR$181 \mathrm{c}-5 \mathrm{p}$ is downregulated in plasma of acute stroke patients [43] and in the hippocampus of rat models of cerebral ischemia/hypoxia [44]. These studies have also shown that miR-181c agomir exacerbates brain ischemia-reperfusion injury [43] and improves cognitive deficits induced by chronic cerebral hypoperfusion [44]. Based on this evidence, we speculate that associations between miR-181c-5p and $A \beta_{1-40}$ are likely instigated by chronic cerebral hypoperfusion and altered neurovascular coupling that lead to increased $A \beta_{1-40}$ levels in aging conditions.

Contrary to what might be expected, especially considering previous evidence linking miR-181c to increased $A \beta_{1-42}$ levels [20] and regulation of $A \beta$ deposition in hippocampal cultures [19], we found no significant associations between serum mir-181c-5p and plasma $A \beta_{1-42}$ concentrations. It may happen that the relationship between mir-181c-5p and $A \beta_{1-42}$ is specifically linked to $\mathrm{AD}$, whereas cerebral changes associated with decreased serum levels of mir-181c-5p is signaling aging-related cerebral vulnerability of unspecified origin. On the other hand, our results differ from those reported in [25]. Siedlecki-Wullich and colleagues [25] showed significant upregulation of miR-92a-3p, miR-181c-5p and miR-210$3 \mathrm{p}$ in the plasma of both MCI and AD subjects. In our study, performed in cognitively normal elderly subjects, we found significant associations between decreased serum expression of miR-181c and increased plasma levels of $A \beta_{1-40}$, deficits in cortical glucose metabolism, and volume reduction of the entorhinal cortex. We speculate that both studies are likely revealing different phenomena (AD vs. aging-related cerebral vulnerability) associated with deregulation of miR-181c-5p. However, it should be mentioned that the two studies employed different blood compartments in miR-181c-5p experiments (serum vs. plasma). Although both plasma and serum are commonly used for extracellular miRNA detection, we selected serum to avoid sample hemolysis and to obtain higher miRNA concentrations [53].

To our knowledge, only a few studies have specifically evaluated the significance of combining serum expression of specific miRNAs and macroscopic brain changes as AD biomarkers. In a seminal work, Cheng and collaborators [54] identified an AD-specific serum 16-miRNA signature that was validated with amyloid-PET imaging, showing high accuracy during early- to mid-AD stage. Further research has revealed that associations between serum miR-223 and magnetic resonance spectroscopy markers of neuronal damage (i.e., $\mathrm{N}$-acetylaspartate and myo-inositol) are able to predict AD severity through inflammatory and apoptosis pathways [55]. A recent study has also shown that lower serum expression of $5 \mathrm{AD}$-related miRNAs (miR-9-5p, miR-29b-3p, miR-34a-5p, miR-125b-5p, and miR-146a-5p) was associated with poorer cognitive functioning and structural and metabolic cortical deficits in aging, indicating that these miRNAs are biologically meaningful in senescence and may play a role as biomarkers of cerebral vulnerability in late life [29]. Since lower miR-181c expression has been tightly related to AD [19], our results linking decreased miR-181c-5p levels and atrophy of entorhinal cortex may be suggestive of accelerated aging and/or reveal certain susceptibility to developing AD. Further research is definitely warranted to clarify if the link between miR181c-5p and entorhinal cortex may serve as a surrogate marker of AD vulnerability.

We have further shown that decreased serum expression of miR-181c-5p was associated with widespread 




Fig. 2 Vertex-wise regression analysis, adjusted by age and sex, to evaluate correlations between serum levels of miR-181c-5p and cortical glucose metabolism, measured with FDG-PET. a Significant patterns of correlations were represented on inflated cortical surfaces ( $L$, left; $R$, right). Color bars represent corrected $p$-values $(p<0.05)$ using a hierarchical approach based on sequential statistical thresholding [35]. b Significant patterns of correlations displayed on flattened cortical surfaces. Squares with colored borders limit the location of significant regional changes. $\mathbf{c}$ The surface of the square was zoomed on flattened cortical maps displaying cytoarchitectonic delimitation of affected regions [36-40]

cortical hypometabolism rather than with patterns of cortical thinning. Many studies have confirmed that reduced FDG-PET brain metabolism precedes cortical atrophy in early $\mathrm{AD}[56,57]$. In the present study, hypometabolic cortical regions that correlated with lower levels of miR-181c-5p are reminiscent of those that predict conversion to dementia in asymptomatic subjects (i.e., temporo-parietal and prefrontal cortex) [58], which may indicate certain susceptibility to developing $\mathrm{AD}$ in individuals with decreased miR-181c-5p. Moreover, previous studies have found that oxygen-glucose deprivation downregulates miR-181c expression in primary microglia by targeting Toll-like receptor 4 (TLR4), suggesting that miR-181c may also be involved in the regulation of the inflammatory response to hypoxic injuries [59]. Therefore, we speculate that, initially, early 
soluble $A \beta$ oligomers lead to miR-181c deregulation [19] and subsequently lower miR-181c expression levels contribute to $\mathrm{AD}$ progression through deregulation of inflammatory response to hypoxia [59]. In this context, a deficient cortical glucose metabolism may reinforce multiple feedback loops of disease progression [60] accelerating the emergence of cognitive impairment. Alternatively, deregulation of miR-181c-5p may instigate the pathogenesis of $\mathrm{AD}$ by targeting specific proteins at the post-transcriptional level. This hypothesis is supported by experiments showing that low expression of miR$181 \mathrm{c}$ in the hippocampus of senescence-accelerated mice increases the collapsin response mediator protein-2 [61], a microtubule associated protein whose phosphorylation is required for $A \beta$-induced memory deficits [62]. Future experiments are required to determine whether miR181c-5p deregulation is the cause or the consequence of cerebral vulnerability in aging and/or AD pathogenesis.

Accumulated research has highlighted the role played by serum miRNAs as potential biomarkers for AD [12-18]. In one of these studies, authors showed a significant upregulation of miR-455-3p in serum of AD patients, which was validated in postmortem $\mathrm{AD}$ brain tissues, $\mathrm{AD}$ cell lines, and in the cerebral cortex of amyloid precursor protein transgenic mice [18]. These findings have been recently extended to $\mathrm{AD}$ fibroblasts cells [63]. miR-455-3p was further related to genes directly associated with $\mathrm{AD}$ pathogenesis, revealing possible molecular links between miR-455-3p and $\mathrm{AD}$ progression $[18,63]$. While these findings strongly suggest that miR-455-3p may be a potential serum biomarker for $\mathrm{AD}$, its remains to be established its role in detecting asymptomatic subjects at risk for developing $\mathrm{AD}$.

The present study is subject to certain limitations that should be mentioned. Participants were clinically normal elderly subjects. Therefore, our results do not allow establishing the prognostic value for the development of AD. Follow-up studies are necessary to determine to what extent lower serum expression of miR-181c-5p is a biomarker of $\mathrm{AD}$, and whether cerebral markers reported here contribute to validate that prediction. Moreover, subjects lacked surrogate biomarkers to confirm AD pathology (e.g., CSF or PET-based biomarkers), impeding to determine the risk for $\mathrm{AD}$. Consequently, these findings should be replicated in a well-characterized cohort with $\mathrm{AD}$ biomarkers and compared with other neurodegenerative diseases (e.g., vascular dementia, Parkinson disease, fronto-temporal dementia) to further establish their specificity in $\mathrm{AD}$.

\section{Conclusions}

In summary, our findings indicate that lower serum expression of miR-181c-5p is associated with higher plasma concentrations of $A \beta_{1-40}$, widespread patterns of cortical hypometabolism, and volume loss of the entorhinal cortex in normal elderly subjects These results highlight the value of serum miR-181c-5p at determining cerebral vulnerability in normal aging.

\section{Supplementary information}

Supplementary information accompanies this paper at https://doi.org/10. 1186/s40035-019-0174-8

Additional file 1: Figure S1. Mean/standard deviation of cycle threshold (Ct) values (3 replicas) of mir-181c-5p (gray circles) and cel-miR39-3p (open circles) for each study participant.

Additional file 2: Figure S2. Correlations segregated by sex between serum levels of miR-181c-5p and plasma A $\beta$ levels (A, B), and volume of $A D$-related brain regions $(C-H)$. Variables included in the scatter plots correspond to the standardized residuals obtained from linear regression analyses adjusted by age, $A \beta_{1-42}$ (in the case of $A \beta_{1-40}$ ), $A \beta_{1-40}$ (in the case of $A \beta_{1-42}$ ), and ICV (in the case of left and right cerebral regions). Note that only correlations between serum mir-181c-5p and plasma $A \beta_{1-40}$ levels yielded significant in women.

\section{Abbreviations}

AD: Alzheimer's disease; $A \beta$ : Amyloid- $\beta$; BNT: Boston Naming Test; CDNA: Complementary deoxyribonucleic acid; CDR: Clinical Dementia Rating; CSF: Cerebrospinal fluid; Ct: Cycle threshold; FCSRT: Free and Cued Selective Reminding Test; FDG: 2-[18F]fluoro-2-deoxy-D-glucose; MFQ: Memory Functioning Questionnaire; miRNA: Micro ribonucleic acid; MMSE: Mini Mental State Examination; MRI: Magnetic resonance imaging; mRNA: Messenger ribonucleic acid; PET: Positron emission tomography; qRTPCR: Quantitative real-time Polymerase Chain Reaction; ROI: Region of interest; RT: Reverse transcription; TLR4: Toll-like receptor 4; TOL: Tower of London; WM: White matter

\section{Acknowledgements}

Not applicable.

\section{Authors' contributions}

MMC, MA and JLC contributed to data acquisition, data analysis, and preparation of tables and figures. MA and JLC contributed to the design and concept of the study. JLC wrote the manuscript. All authors critically reviewed and approved the final manuscript.

\section{Funding}

This work was supported by research grants from the Spanish Ministry of Economy and Competitiveness (SAF2017-85310-R to JLC, PSI2017-85311-P to MA); the Regional Ministry of Innovation, Science and Enterprise, Junta de Andalucía (P12-CTS-2327 to JLC); the International Center on Aging CENIEPOCTEP (0348_CIE_6_E to MA); and CIBERNED (CB06/05/1111 to JLC).

\section{Availability of data and materials}

The datasets used and analyzed in the current study are available from the corresponding author on reasonable request.

\section{Ethics approval and consent to participate}

The study was approved by the Ethical Committee for Human Research at the Pablo de Olavide University, and all individuals gave written informed consent to participate.

\section{Consent for publication}

Not applicable.

\section{Competing interests}

The authors declare that they have no competing interests.

Received: 12 July 2019 Accepted: 25 September 2019

Published online: 04 November 2019

\section{References}

1. Kirkwood TB, Austad SN. Why do we age? Nature. 2000;408:233-8. 
2. Niccoli T, Partridge L. Ageing as a risk factor for disease. Curr Biol. 2012;22:R741-52.

3. Baur JA, Pearson KJ, Price NL, Jamieson HA, Lerin C, Kalra A, et al. Resveratrol improves health and survival of mice on a high-calorie diet. Nature. 2006;444:337-42.

4. Colman RJ, Anderson RM, Johnson SC, Kastman EK, Kosmatka KJ, Beasley $\mathrm{TM}$, et al. Caloric restriction delays disease onset and mortality in rhesus monkeys. Science. 2009;325:201-4.

5. Wilkinson JE, Burmeister L, Brooks SV, Chan CC, Friedline S, Harrison DE, et al. Rapamycin slows aging in mice. Aging Cell. 2012;11:675-82.

6. Blagosklonny MV. Disease or not, aging is easily treatable. Aging (Albany NY). 2018;10:3067-78.

7. Partridge L. Intervening in ageing to prevent the diseases of ageing. Trends Endocrinol Metab. 2014;25:555-7.

8. Bartel DP. MicroRNAs: genomics, biogenesis, mechanism, and function. Cell. 2004:116:281-97.

9. Mitchell PS, Parkin RK, Kroh EM, Fritz BR, Wyman SK, Pogosova-Agadjanyan EL, et al. Circulating microRNAs as stable blood-based markers for cancer detection. Proc Natl Acad Sci U S A. 2008;105:10513-8.

10. Turchinovich A, Weiz L, Langheinz A, Burwinkel B. Characterization of extracellular circulating microRNA. Nucleic Acids Res. 2011;39:7223-33.

11. Dehghani R, Rahmani F, Rezaei N. MicroRNA in Alzheimer's disease revisited: implications for major neuropathological mechanisms. Rev Neurosci. 2018; 29:161-82.

12. Tan L, Yu JT, Liu QY, Tan MS, Zhang W, Hu N, et al. Circulating miR-125b as a biomarker of Alzheimer's disease. J Neurol Sci. 2014;336:52-6.

13. Nagaraj S, Laskowska-Kaszub K, Dębski KJ, Wojsiat J, Dąbrowski M, Gabryelewicz T, et al. Profile of 6 microRNA in blood plasma distinguish early stage Alzheimer's disease patients from non-demented subjects. Oncotarget. 2017;8:16122-43.

14. Dong H, Li J, Huang L, Chen X, Li D, Wang T, et al. Serum microRNA profiles serve as novel biomarkers for the diagnosis of Alzheimer's disease. Dis Markers. 2015;2015:625659.

15. Galimberti D, Villa C, Fenoglio C, Serpente M, Ghezzi L, Cioffi SM, et al. Circulating miRNAs as potential biomarkers in Alzheimer's disease. J Alzheimers Dis. 2014;42:1261-7.

16. Leidinger $P$, Backes $C$, Deutscher S, Schmitt K, Mueller SC, Frese K, et al. A blood based 12-miRNA signature of Alzheimer disease patients. Genome Biol. 2013;14:R78.

17. Geekiyanage H, Jicha GA, Nelson PT, Chan C. Blood serum miRNA: noninvasive biomarkers for Alzheimer's disease. Exp Neurol. 2012;235:491-6.

18. Kumar S, Vijayan M, Reddy PH. MicroRNA-455-3p as a potential peripheral biomarker for Alzheimer's disease. Hum Mol Genet. 2017;26:3808-22.

19. Schonrock N, Humphreys DT, Preiss T, Götz J. Target gene repression mediated by miRNAs miR-181C and miR-9 both of which are downregulated by amyloid- $\beta$. J Mol Neurosci. 2012;46:324-35.

20. Schonrock N, Ke YD, Humphreys D, Staufenbiel M, Ittner LM, Preiss T, et al. Neuronal microRNA deregulation in response to Alzheimer's disease amyloid-beta. PLoS One. 2010;5(6):e11070

21. Cogswell JP, Ward J, Taylor IA, Waters M, Shi Y, Cannon B, et al. Identification of miRNA changes in Alzheimer's disease brain and CSF yields putative biomarkers and insights into disease pathways. J Alzheimers Dis. 2008;14:27-41.

22. Geekiyanage $H$, Chan $C$. MicroRNA-137/181c regulates serine palmitoyltransferase and in turn amyloid beta, novel targets in sporadic Alzheimer's disease. J Neurosci. 2011;31:14820-30.

23. Nunez-Iglesias J, Liu CC, Morgan TE, Finch CE, Zhou XJ. Joint genome-wide profiling of miRNA and mRNA expression in Alzheimer's disease cortex reveals altered miRNA regulation. PLoS One. 2010;52(2):e8898.

24. Guo R, Fan G, Zhang J, Wu C, Du Y, Ye H, et al. A 9-microRNA signature in serum serves as a noninvasive biomarker in early diagnosis of Alzheimer's disease. J Alzheimers Dis. 2017:60:1365-77.

25. Siedlecki-Wullich D, Català-Solsona J, Fábregas C, Hernández I, Clarimon J, Lleó A, et al. Altered microRNAs related to synaptic function as potential plasma biomarkers for Alzheimer's disease. Alzheimers Res Ther. 2019;11(1):46.

26. Böhm P, Peña-Casanova J, Aguilar M, Hernandez G, Sol JM, Blesa R, et al. Clinical validity and utility of the interview for deterioration of daily living in dementia for Spanish-speaking communities. Int Psychogeriatr. 1998;10:261-70.

27. Yesavage JA, Brink TL, Rose TL, Lum O. Development and validation of a geriatric depression scale: a preliminary report. J Psychiat Res. 1983:17:37-49.

28. Livak KJ, Schmittgen TD. Analysis of relative gene expression data using real-time quantitative PCR and the 2(-Delta Delta $C(T))$ method. Methods. 2001;25:402-8.
29. Maldonado-Lasuncion I, Atienza M, Sanchez-Espinosa MP, Cantero JL. Aging-related changes in cognition and cortical integrity are associated with serum expression of candidate microRNAs for Alzheimer disease. Cereb Cortex. 2019;29(10):4426-37.

30. Fischl B, Dale AM. Measuring the thickness of the human cerebral cortex from magnetic resonance images. Proc Natl Acad Sci U S A. 2000;97:11050-5.

31. Bernal-Rusiel $J \mathrm{~L}$, Atienza M, Cantero JL. Detection of focal changes in human cortical thickness: spherical wavelets versus Gaussian smoothing. Neuroimage. 2008;41:1278-92.

32. Park HJ, Lee JD, Chun JW, Seok JH, Yun M, Oh MK, et al. Cortical surfacebased analysis of 18F-FDG PET: measured metabolic abnormalities in schizophrenia are affected by cortical structural abnormalities. Neuroimage. 2006:31:1434-44.

33. Destrieux C, Fischl B, Dale A, Halgren E. Automatic parcellation of human cortical gyri and sulci using standard anatomical nomenclature. Neuroimage. 2010;53:1-15

34. Braak H, Braak E. Neuropathological stageing of Alzheimer-related changes. Acta Neuropathol. 1991;82:239-59.

35. Bernal-Rusiel JL, Atienza M, Cantero JL. Determining the optimal level of smoothing in cortical thickness analysis: a hierarchical approach based on sequential statistical thresholding. Neuroimage. 2010;52:158-71.

36. Bludau S, Eickhoff SB, Mohlberg H, Caspers S, Laird AR, Fox PT, et al. Cytoarchitecture, probability maps and functions of the human frontal pole. Neuroimage. 2014;93:260-75

37. Ding SL, Van Hoesen GW, Cassell MD, Poremba A. Parcellation of human temporal polar cortex: a combined analysis of multiple cytoarchitectonic, chemoarchitectonic, and phthological markers. J Comp Neurol. 2009;514: 595-623.

38. McDonald B, Highley JR, Walker MA, Herron BM, Cooper SJ, Esiri MM, et al. Anomalous asymmetry of fusiform and parahipocampal gyrus gray matter in schizophrenia: a postmortem study. Am J Psychiatry. 2000;157:40-7.

39. Malikovic A, Amunts K, Schleicher A, Mohlberg H, Kujovic M, PalomeroGallagher N, et al. Cytoarchitecture of the human lateral occipital cortex: mapping of two extrastriate areas hOc4la and hOc4lp. Brain Struct Funct. 2016;221:1877-97

40. Scheperjans F, Eickhoff SB, Hömke H, Mohlberg K, Hermann K, Amunts K, et al. Probabilistic maps, morphometry, and variability of citoarchitectonic areas in the human superior parietal cortex. Cereb Cortex. 2008;18:2141-57.

41. Hampel H, O'Bryant SE, Molinuevo JL, Zetterberg H, Masters CL, Lista S, et al. Blood-based biomarkers for Alzheimer disease: mapping the road to the clinic. Nat Rev Neurol. 2018;14:639-52.

42. Kumar S, Reddy PH. Are circulating microRNAs peripheral biomarkers for Alzheimer's disease? Biochim Biophys Acta. 1862;2016:1617-27.

43. Ma Q, Zhao H, Tao Z, Wang R, Liu P, Han Z, et al. MicroRNA-181C exacerbates brain injury in acute ischemic stroke. Aging Dis. 2016;7:705-14.

44. Fang C, Li Q, Min G, Liu M, Cui J, Sun J, et al. MicroRNA-181C ameliorates cognitive impairment induced by chronic cerebral hypoperfusion in rats. Mol Neurobiol. 2017;54:8370-85.

45. Scheuner D, Eckman C, Jensen M, Song X, Citron M, Suzuki N, et al. Secreted amyloid beta-protein similar to that in the senile plaques of Alzheimer's disease is increased in vivo by the presenilin 1 and 2 and APP mutations linked to familial Alzheimer's disease. Nat Med. 1996;2:864-70.

46. Kosaka T, Imagawa M, Seki K, Arai H, Sasaki H, Tsuji S, et al. The beta APP717 Alzheimer mutation increases the percentage of plasma amyloid-beta protein ending at A beta42(43). Neurology. 1997:48:741-5.

47. Schupf N, Patel B, Pang D, Zigman WB, Silverman W, Mehta PD, et al. Elevated plasma beta-amyloid peptide Abeta (42) levels, incident dementia, and mortality in Down syndrome. Arch Neurol. 2007;64:1007-13.

48. Head E, Doran E, Nistor M, Hill M, Schmitt FA, Haier RJ, et al. Plasma amyloid-beta as a function of age, level of intellectual disability, and presence of dementia in Down syndrome. J Alzheimers Dis. 2011;23: 399-409.

49. Ertekin-Taner N, Younkin LH, Yager DM, Parfitt F, Baker MC, Asthana S, et al. Plasma amyloid beta protein is elevated in late-onset Alzheimer disease families. Neurology. 2008;70:596-606.

50. Llado-Saz S, Atienza M, Cantero JL. Increased levels of plasma amyloid-beta are related to cortical thinning and cognitive decline in cognitively normal elderly subjects. Neurobiol Aging. 2015;36:2791-7.

51. van Leijsen EMC, Kuiperij HB, Kersten I, Bergkamp MI, van Uden IWM,

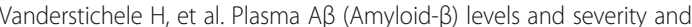
progression of small vessel disease. Stroke. 2018;49:884-90. 
52. Gomis M, Sobrino T, Ois A, Millán M, Rodríguez-Campello A, Pérez de la Ossa $\mathrm{N}$, et al. Plasma beta-amyloid 1-40 is associated with the diffuse small vessel disease subtype. Stroke. 2009;40:3197-201.

53. Wang K, Yuan Y, Cho JH, McClarty S, Baxter D, Galas DJ. Comparing the MicroRNA spectrum between serum and plasma. PLoS One. 2012;7(7):e41561.

54. Cheng L, Doecke JD, Sharples RA, Villemagne VL, Fowler CJ, Rembach A, et al. Prognostic serum miRNA biomarkers associated with Alzheimer's disease shows concordance with neuropsychological and neuroimaging assessment. Mol Psychiatry. 2015;20:1188-96.

55. Wei $H, X u Y, X u$ W, Zhou Q, Chen $Q$, Yang $M$, et al. Serum exosomal miR223 serves as a potential diagnostic and prognostic biomarker for dementia. Neuroscience. 2018;379:167-76

56. Kim EJ, Cho SS, Jeong Y, Park KC, Kang SJ, Kang E, et al. Glucose metabolism in early onset versus late onset Alzheimer's disease: an SPM analysis of 120 patients. Brain. 2005:128:1790-801.

57. Mosconi L, Mistur R, Switalski R, Tsui WH, Glodzik L, Li Y, et al. FDG-PET changes in brain glucose metabolism from normal cognition to pathologically verified Alzheimer's disease. Eur J Nucl Med Mol Imaging. 2009:36:811-22.

58. Ewers M, Brendel M, Rizk-Jackson A, Rominger A, Bartenstein P, Schuff N, et al. Reduced FDG-PET brain metabolism and executive function predict clinical progression in elderly healthy subjects. Neuroimage Clin. 2013:4:45-52.

59. Zhang L, Li YJ, Wu XY, Hong Z, Wei WS. MicroRNA-181c negatively regulates the inflammatory response in oxygen-glucose-deprived microglia by targeting toll-like receptor 4. J Neurochem. 2015;132:713-23.

60. Zilberter Y, Zilberter M. The vicious circle of hypometabolism in neurodegenerative diseases: ways and mechanisms of metabolic correction. J Neurosci Res. 2017:95:2217-35.

61. Zhou H, Zhang R, Lu K, Yu W, Xie B, Cui D, et al. Deregulation of miRNA181c potentially contributes to the pathogenesis of AD by targeting collapsin response mediator protein 2 in mice. J Neurol Sci. 2016;367:3-10

62. Isono T, Yamashita N, Obara M, Araki T, Nakamura F, Kamiya Y, et al. Amyloid- $\beta_{25-35}$ induces impairment of cognitive function and long-term potentiation through phosphorylation of collapsin response mediator protein 2. Neurosci Res. 2013;77:180-5.

63. Kumar S, Reddy PH. MicroRNA-455-3p as a potential biomarker for Alzheimer's disease: an update. Front Aging Neurosci. 2018;10:41.

Ready to submit your research? Choose BMC and benefit from:

- fast, convenient online submission

- thorough peer review by experienced researchers in your field

- rapid publication on acceptance

- support for research data, including large and complex data types

- gold Open Access which fosters wider collaboration and increased citations

- maximum visibility for your research: over $100 \mathrm{M}$ website views per year

At $\mathrm{BMC}$, research is always in progress.

Learn more biomedcentral.com/submissions 